\title{
Facilitando a Distribuição Segura de Dados de Contexto em Aplicações de Internet das Coisas Móveis
}

\author{
André Luiz A. Cardoso ${ }^{1}$, Thiago Wallass N. Mendes $^{1}$, Francisco José S. Silva ${ }^{1}$ \\ Ariel S. Teles ${ }^{1}$ \\ ${ }^{1}$ Laboratório de Sistemas Distribuídos Inteligentes \\ Universidade Federal do Maranhão (UFMA) \\ São Luís - MA - Brasil \\ \{andre.cardoso, thiago.wallass, fssilva, ariel\}alsdi.ufma.br
}

\begin{abstract}
Internet of Mobile Things (IoMT) applications have the potential to solve many problems. Health and wellness, logistics and smart cities are some areas that can benefit from it. A frequent approach to IoMT solutions involves the use of middleware, which aims to serve as a basis for developing applications. In this paper, we propose the addition, for optional use, of a security layer to the M-Hub/CDDL middleware. The main goal of the proposed solution is to guarantee secure communication, authentication and access control. By performing a performance evaluation, the computational cost regarding processing and memory consumption in the mobile side of the solution was identified due to the use of the layer.
\end{abstract}

Resumo. Aplicações de Internet das Coisas Móveis (IoMT) possuem potencial para solucionar diversos problemas. Saúde e bem estar, logística e cidades inteligentes são alguns domínios que podem se beneficiar disso. Uma abordagem frequente para soluções de IoMT envolve o uso de middleware, o qual visa servir de base para o desenvolvimento de aplicações. Este trabalho propõe a adição, para uso opcional, de uma camada de segurança ao middleware M-Hub/CDDL. O objetivo da solução proposta é garantir a comunicação segura, autenticação e controle de acesso. Através de uma avaliação de desempenho, identificou-se o custo computacional de processamento e consumo de memória no lado móvel da solução devido ao uso da camada.

\section{Introdução}

O paradigma de Internet das Coisas (IoT) reúne diversos conceitos e tecnologias, tais como computação ubíqua e pervasiva, e tecnologias de sensoriamento e comunicação. Dessa forma, dispositivos equipados com sensores e atuadores (e.g., smartphones) podem se interconectar de maneira que haja uma interação contínua entre o mundo real e o digital [Borgia 2014]. Sistemas IoT permitem que objetos sejam capazes de interagir entre si, atuem de acordo com as suas interpretações e troquem informações com pessoas. Estes objetos, sendo físicos ou digitais, constituem a base da IoT e são chamados de Objetos Inteligentes [Kortuem et al. 2009].

A Internet das Coisas Móveis (IoMT) é uma parte da IoT em que tanto os objetos quanto os gateways IoT podem ser movidos ou moverem-se de forma autônoma, permanecendo acessíveis e controláveis através da Internet [Talavera et al. 2015]. A 
IoMT já está inserida de maneira significativa em diversos domínios de aplicação, tais como cidades inteligentes, segurança publica, logística, saúde e bem estar [Endler and e Silva 2018]. A heterogeneidade dos dispositivos de IoT é um problema recorrente, e uma possível solução consiste em utilizar soluções de middleware.

Um middleware de IoT pode ser descrito como um software que atua como intermediário entre os dispositivos IoT e as aplicações [Ngu et al. 2016]. Através da abstração de diferentes tecnologias, um middleware pode oferecer uma interface mais simples para o programador desenvolver suas soluções. O M-Hub/CDDL [Gomes et al. 2017] é uma dessas soluções de middleware, o qual combina um gateway móvel com uma camada de distribuição de dados e visa facilitar o desenvolvimento de aplicações de IoMT [Gomes et al. 2017]. Como exemplo de aplicação, considere um paciente que necessita de acompanhamento constante dos sinais vitais, em que os dados são coletados por sensores de dispositivos vestíveis. O médico monitora os dados à distância verificando a ocorrência de alguma anormalidade. Esses dados de saúde são especialmente sensíveis, sendo necessário, portanto, garantir a segurança na comunicação e no acesso a eles.

Este estudo propõe a adição de uma camada de segurança ao middleware MHub/CDDL, oferecendo um canal seguro de comunicação, mecanismos de autenticação e controle de acesso. A camada de segurança faz o gerenciamento dos elementos necessários para o funcionamento dos mecanismos propostos. Dessa maneira, torna-se possível a distribuição de dados de forma segura entre instâncias do M-Hub/CDDL. Além disso, é possível realizar o ajuste da privacidade na distribuição de dados, combinando o controle de acesso, componente da camada de segurança proposta por esse artigo, com os filtros já existentes no middleware.

O restante do artigo está organizado da seguinte forma. A Seção 2 introduz o middleware M-Hub/CDDL, necessário para o entendimento da solução proposta. A Seção 3 aborda a solução proposta. A Seção 4 apresenta uma avaliação de desempenho que compara o custo computacional com e sem a utilização dos recursos de segurança. A Seção 5 discute os resultados e compara a solução com trabalhos relacionados. Por fim, a Seção 6 conclui o trabalho e apresenta as perspectivas de trabalhos futuros.

\section{Fundamentação Teórica}

\subsection{O middleware M-Hub/CDDL}

O M-Hub/CDDL é um middleware com recursos para a aquisição, processamento e distribuição de dados de contexto, oferecendo suporte para o desenvolvimento de aplicações de IoT/IoMT [Gomes et al. 2017]. O M-Hub (Mobile Hub) atua como gateway móvel para a aquisição dos dados, enquanto o CDDL (Context Data Distribution Layer) é o responsável pela distribuição dos dados com suporte de qualidade de contexto [Manzoor et al. 2014].

O middleware suporta diferentes plataformas. O M-Hub atua em dispositivos móveis que utilizam o sistema operacional Android, já o CDDL possui três tipos de clientes: móveis (Android), desktop e web. Dessa forma, smartphones podem transmitir dados para instâncias móveis do M-Hub/CDDL ou para instâncias que funcionam na nuvem. 


\subsection{Mobile Hub}

O M-Hub atua como intermediário entre sensores, atuadores e a conexão com servidores na nuvem (i.e., via CDDL). Implementada no M-Hub, a interface Short-range Sensing, Presence and Actuation (S2PA), atua como protocolo para comunicação de curto alcance com objetos móveis [Talavera et al. 2015]. Atualmente a S2PA implementa comunicação via Bluetooth 4.0 (BLE) e Bluetooth clássico. Além disso, também fornece acesso aos dados provenientes dos sensores internos de smartphones Android. Vários dispositivos vestíveis, veículos, sensores e atuadores podem ter seus dados coletados e receber comandos de atuação através do M-Hub.

\subsection{CDDL}

A camada de distribuição de dados de contexto (i.e., CDDL) pode ser utilizada de maneira integrada ao M-Hub para transmissão e enriquecimento dos dados coletados. O CDDL utiliza o MQTT ${ }^{1}$ como protocolo de comunicação de dados, seja em conexões locais ou remotas [Gomes et al. 2017]. A Figura 1 exibe a arquitetura atual do M-Hub/CDDL.

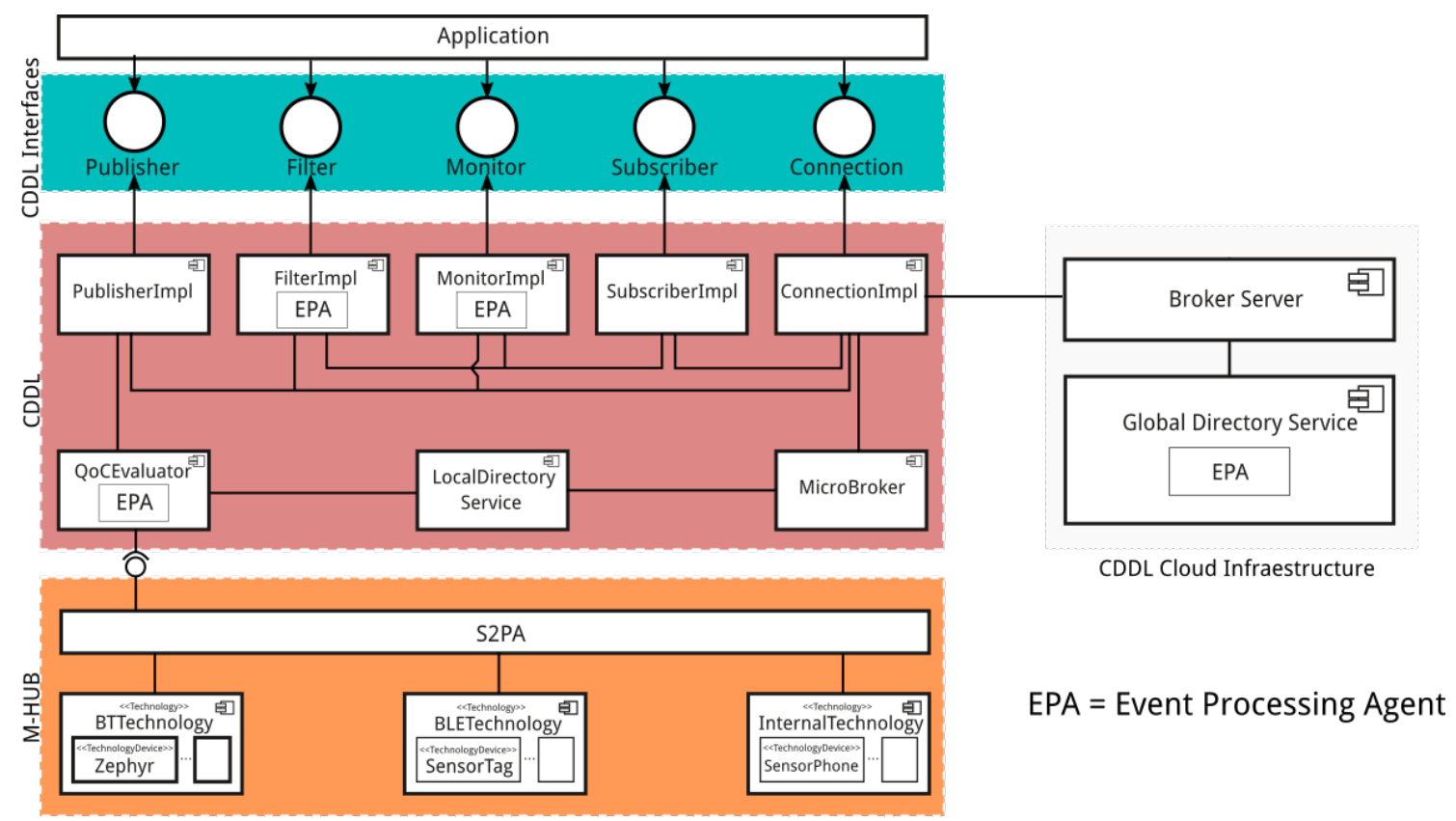

Figura 1. Arquitetura atual do M-Hub/CDDL [Gomes et al. 2017]

O M-Hub, através do S2PA, abstrai diferentes tecnologias permitindo assim a coleta de dados provenientes de objetos inteligentes. Uma vez coletados, o componente QoCEvaluator do CDDL é responsável por enriquecer os dados com informações de contexto tais como: latitude, longitude, acurácia, e timestamp. Os componentes responsáveis pelo transporte dos dados são o Microbroker e o Conection Impl.

O Microbroker encapsula uma versão modificada de um broker MQTT, já o componente Conection Impl encapsula um cliente MQTT, que pode ser utilizado para publicar/subscrever em tópicos. Por fim, o CDDL oferece interfaces que são utilizadas por aplicações e, através delas, pode-se consumir os recursos do CDDL de maneira simples.

\footnotetext{
${ }^{1}$ http://mqtt.org/
} 


\subsection{Problemática Abordada}

O protocolo de comunicação MQTT, utilizado pelo M-Hub/CDDL, já oferece suporte para mecanismos de segurança. A nível de transporte, tem-se o Transport Layer Security (TLS), que objetiva prover segurança de ponta a ponta contra ataques ativos do tipo man in the middle [Krawczyk et al. 2013]. Além de um canal de comunicação seguro, o TLS também pode impedir usuários não autenticados de estabelecerem conexão. O controle de acesso pode variar de acordo com o broker, mas uma forma comum é através de uma lista que armazena os tópicos que os usuários podem acessar. Atualmente, o M-Hub/CDDL não oferece meios para aproveitar-se de tais recursos.

\section{Solução Proposta: um Serviço de Segurança}

A solução proposta, chamada de Security Service (SS), acrescenta uma camada de segurança ao M-Hub/CDDL. Esta camada fornece meios para o estabelecimento de um canal seguro de comunicação, mecanismos de autenticação e controle de acesso. Para o funcionamento desses recursos, é necessário possuir os seguintes itens: chaves criptográficas, certificados digitais e lista de controle de acesso. Tais elementos podem ser gerados e armazenados utilizando o próprio SS. E para o desenvolvimento da camada de segurança, a seguinte política foi considerada:

- Uma instância do M-Hub/CDDL deve manter dois certificado digitais, um para o usuário e outro para a autoridade certificadora;

- A troca de mensagens deve utilizar criptografia;

- A autenticação deve ser mútua em uma conexão;

- O controle de acesso deve, por padrão, recusar todas as mensagens, a não ser que a permissão seja garantida pelo usuário;

- Por fim, o uso do middleware com os recursos de segurança deve ser opcional, permitindo a não utilização do SS.

\subsection{Gerência da Segurança}

O SS oferece meios para: gerar chaves criptográficas, gerar requisição de assinatura de certificado (Certificate Signing Request - CSR), importar o certificado da autoridade certificadora confiável e importar o certificado do usuário do CDDL assinado pela mesma autoridade. Estes métodos são disponibilizados através de uma interface, e apresentados na Figura 2. Através deles, torna-se possível configurar o M-Hub/CDDL para que possa ser utilizado no modo seguro.

\begin{tabular}{|c|}
\hline $\begin{array}{c}\quad\langle\langle\text { Interface }\rangle\rangle \\
\text { Security Service Interface }\end{array}$ \\
\hline $\begin{array}{l}\text { + generateKeyPair(): Void } \\
\text { + generateCSR(String CN, String OU, String O, String L, String S, String C): Void } \\
\text { + setCaCertificate(String filePath): Void } \\
\text { + setCertificate(String filePath): Void } \\
\text { + getCertificate(): Certificate } \\
\text { + getCACertificate(): Certificate }\end{array}$ \\
\hline
\end{tabular}

Figura 2. Métodos da interface relacionados ao gerenciamento de segurança.

Para armazenar as chaves e certificados de maneira segura, levou-se em conta o tipo de cliente CDDL. Para as instâncias móveis do M-Hub/CDDL, utilizou-se o armazenamento interno do Android. Nesse caso, o sistema operacional garante que apenas a 
aplicação pode acessar os arquivos que ela gerou. Para as instâncias desktop, foi utilizado uma keystore do tipo PKCS12 [Focardi et al. 2018] com senha.

\subsection{Canal Seguro de Comunicação e Autenticação}

Uma vez que o MQTT possui suporte ao TLS, ele foi utilizado para garantir o canal seguro de comunicação com autenticação mútua (cliente e broker). Os componentes, pertencentes ao M-Hub/CDDL, cuja responsabilidade é estabelecer conexões, são respectivamente o Microbroker e o Connection. Esses componentes são acessados por meio da interface do CDDL. Para tornar o uso da segurança opcional, adicionou-se novos métodos à interface Connection e à classe Microbroker (Figura 3).
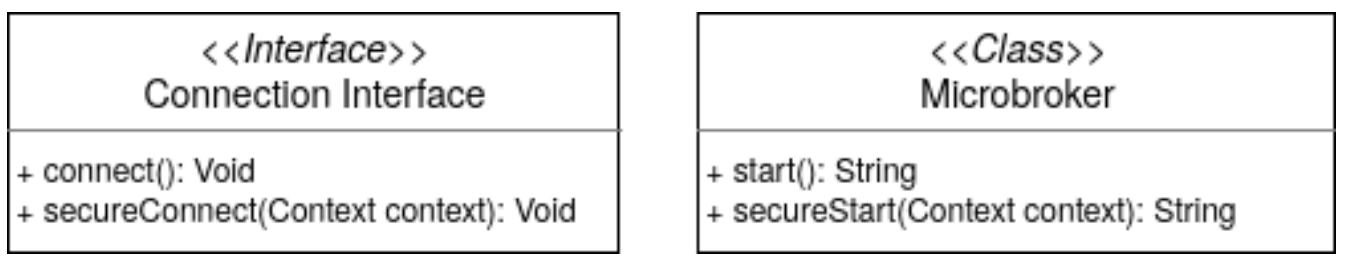

Figura 3. Componentes com os novos métodos de inicialização.

Com essas alterações, é possível iniciar o broker local e se conectar de forma segura. Ao serem chamados, os novos métodos (i.e., secureConnect e securestart) automaticamente buscam as chaves e certificados anteriormente configurados e os utilizam na construção do contexto SSL (Secure Sockets Layer) ${ }^{2}$ para a comunicação.

\subsection{Controle de Acesso}

O CDDL possui uma estrutura predefinida de tópicos baseada em serviços, o usuário apenas define quais serviços quer publicar/sobrescrever dados e o CDDL determina os tópicos automaticamente. Para gerenciar o acesso, é necessário criar regras que determinem quais serviços podem ser acessados. Através de sua interface, o SS oferece métodos para geração de regras. A Figura 4 exibe os métodos disponibilizados pelo SS para adicionar e remover as regras.

\begin{tabular}{|c|}
\hline $\begin{array}{c}\langle\text { Interface }\rangle \\
\text { Security Service Interface }\end{array}$ \\
\hline+ grantPermissionByServiceName(String clientID, String serviceName, String permission): Void \\
+ removePermissionByServiceName(String clientID, String serviceName, String permission): Void
\end{tabular}

Figura 4. Modificação dos componentes Connection e Microbroker.

Utilizando os métodos acima, é possível conceder e revogar permissões para os clientes. Ao serem utilizados, regras são armazenadas pelo SS em um arquivo. Tais regras são geradas de acordo com o seguinte padrão: "ClientID tópico permissão". A Figura 5 exemplifica o resultado obtido ao se adicionar regras de controle de acesso.

O Microbroker oferece uma interface autorizadora que possui dois métodos: canRead e canWrite. Esses métodos são chamados automaticamente no broker

\footnotetext{
${ }^{2}$ https://docs.oracle.com/javase/7/docs/api/javax/net/ssl/SSLContext.html
} 


\begin{tabular}{|c|}
\hline Regras \\
\hline $\begin{array}{l}\text { usuarioXYZ } \\
\text { usuarioXYZ }\end{array}$ mhub/t/service_topic/sensorXYZ read $\mathrm{m}$ m $\mathrm{Y} /$ service_topic/sensorXYZ write \\
\hline
\end{tabular}

Figura 5. Exemplo de regras geradas utilizando o Security Service.

quando um cliente tenta publicar ou subscrever em tópicos. Assim, implementou-se esta interface autorizadora e seus métodos de forma que busquem e utilizem regras armazenadas no SS para permitir ou bloquear o acesso.

\section{Avaliação de Desempenho}

Para avaliar a solução proposta, foi utilizado uma aplicação para gestão de presença e encontros de pessoas em ambientes fechados. Esta aplicação utiliza um smartphone com o M-Hub/CDDL para detectar beacons Bluetooth Low Energy (BLE) e transmitir dados para um servidor executando uma instância desktop do CDDL. A aplicação móvel utiliza o Mi crobroker para coletar os dados do ambiente e uma conexão remota para enviar a notificação ao servidor. Esta aplicação trabalha de maneira intensa, sendo capaz de detectar aproximadamente 5 objetos por segundo. Por ser a o componente do M-Hub/CDDL com restrição de recursos, avaliou-se a instância móvel objetivando medir o desempenho da aplicação com e sem a utilização da camada de segurança.

Para esta avaliação, utilizou-se um smartphone do modelo Moto G8 Plus, o qual possui as seguintes especificações: sistema operacional Android 9, processador Qualcomm Snapdragon 665 (4 x 1.8GHz), 4 GB de memória RAM, e 64 GB de armazenamento em disco. A avaliação consistiu em utilizar o Android Profiler ${ }^{3}$ para verificar o consumo total de memória e CPU pela aplicação. O experimento durou 25 minutos e as medições foram verificadas a cada intervalo de 1 minuto. A tela do dispositivo se encontrava apagada durante a avaliação. A Figura 6 exibe os resultados obtidos, o consumo de memória é medido em MB enquanto o processamento é medido em percentual da capacidade total de processamento do dispositivo.
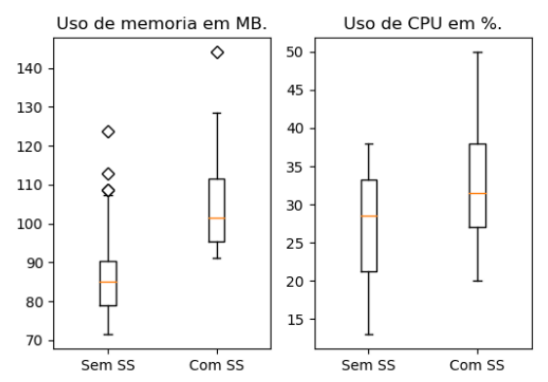

\begin{tabular}{rrrrrr} 
& Média & Mediana & Moda & Desvio Padrão \\
\hline Memória sem SS & 88.415385 & 85.2 & 77.4 & 12.878536 \\
Memória com SS & 105.111538 & 101.6 & 99.5 & 12.959117 \\
CPU sem SS & 27.192308 & 28.5 & 20.0 & 7.076610 \\
CPU com SS & 32.846154 & 31.5 & 27.0 & 8.839128
\end{tabular}

Figura 6. Resultado: custo adicional em memória e processamento.

\section{Discussão}

Para estabelecer uma comunicação segura, deve-se levar em conta as diferentes plataformas que suportam o M-Hub/CDDL. A comunicação entre objetos inteligentes e M-Hub

\footnotetext{
${ }^{3}$ https://developer.android.com/studio/profile/android-profiler
} 
pode ser protegida utilizando os mecanismos que as tecnologias WPAN oferecem (e.g., pareamento Bluetooth). A comunicação segura entre instâncias móveis do M-Hub/CDDL é garantida pelo SS, desde que ambas utilizem a mesma autoridade certificadora. Este princípio também se aplica às instâncias desktop ou a qualquer broker MQTT que ofereça suporte para o TLS. Dessa forma, pode-se assegurar a comunicação em diferentes contextos de aplicação do M-Hub/CDDL.

\subsection{Análise dos Resultados da Avaliação de Desempenho}

Os resultados obtidos demonstram que a camada de segurança é aplicável a um cenário real. A segurança foi implementada para ser um recurso opcional, mas seu uso adiciona algum custo computacional. Em relação a memória, o custo médio sem a utilização da camada foi de 85,2 MB, já utilizando-a houve um aumento de 19,24 \%. Portanto, houve custo extra ao se utilizar a camada de segurança. Outliers ocorreram quando a medição foi feita no momento em que variáveis temporárias foram alocadas em memória para efetuar alguma operação do middleware. Em relação ao processamento, sem a utilização da camada de segurança, o valor médio foi de $28,5 \%$ do poder de processamento do dispositivo. Ao se utilizar a camada de segurança, o valor médio aumenta para 31,5\%. A diferença equivale a um aumento de $10,52 \%$ no custo do processamento. Verificou-se que houve aumento no consumo de ambos recursos ao se utilizar a camada de segurança. Utilizando a camada ou não obtivemos valores de desvio padrão bem próximos, indicando que o custo extra é distribuído uniformemente ao longo da execução. Ao se adicionar uma carga extra ao processamento e memória, devido à criptografia, já era esperado que houvesse aumento nos valores.

\subsection{Comparação com Trabalhos Relacionados}

$\mathrm{Na}$ literatura, existem diversos recursos de segurança para soluções de middleware de IoT [Ngu et al. 2016, Khan and Salah 2018]. Ferreira et al. [Ferreira et al. 2014] apresentaram uma arquitetura de segurança para um middleware transparente. Através dele, pode-se transformar objetos comuns em inteligentes, utilizando mecanismos que garantem privacidade, autenticação, integridade e confidencialidade na troca de dados. Conzon et al. [Conzon et al. 2012] propuseram o VIRTUS, um middleware IoT que utiliza o protocolo XMPP para fornecer comunicações seguras. Além de aproveitar os serviços padrões de segurança do protocolo, o VIRTUS faz uso dos protocolos TLS e SASL para autenticação e criptografia.

Apesar das diferentes tecnologias e objetivos de cada middleware, alguns exibem esforços para proteger a transmissão de dados. A solução apresentada nesse artigo diferencia-se por atuar em um gateway móvel, onde os recursos são restritos, e acrescenta um custo baixo de memória e processamento que é distribuído uniformemente ao longo da execução. Considerando este diferencial, pode-se utilizar um smartphone pessoal, de maneira segura, como gateway em soluções de IoMT. Por funcionar em um middleware publish/subscribe baseado em tópicos, a camada de segurança também estabelece conexão segura com brokers MQTT que suportem o TLS, aumentando sua aplicabilidade.

\section{Conclusão e Trabalhos Futuros}

A camada de segurança, apresentada como proposta de solução deste estudo, oferece meios práticos para que o desenvolvedor possa utilizar o M-Hub/CDDL de forma a garantir um canal de comunicação seguro, além de autenticação e controle de acesso das 
entidades. A avaliação de desempenho da solução proposta mostrou que ela não gera uma sobrecarga excessiva ao dispositivo móvel. Apesar disso, ainda existem melhorias a serem implementadas. Como perspectivas futuras para este trabalho, podemos citar o suporte para múltiplos certificados digitais. Além disso, existe também a possibilidade do uso de blockchain como recurso de segurança, uma vez que essa tecnologia pode ser utilizada para autenticar, autorizar e auditar os dados gerados.

\section{Referências}

Borgia, E. (2014). The internet of things vision: Key features, applications and open issues. Computer Communications, 54:1-31.

Conzon, D., Bolognesi, T., Brizzi, P., Lotito, A., Tomasi, R., and Spirito, M. A. (2012). The virtus middleware: An xmpp based architecture for secure iot communications. In 2012 21st International Conference on Computer Communications and Networks (ICCCN), pages 1-6. IEEE.

Endler, M. and e Silva, F. S. (2018). Past, present and future of the contextnet iomt middleware. Open Journal of Internet Of Things (OJIOT), 4(1):7-23.

Ferreira, H. G. C., de Sousa, R. T., de Deus, F. E. G., and Canedo, E. D. (2014). Proposal of a secure, deployable and transparent middleware for internet of things. In 2014 9th Iberian Conference on Information Systems and Technologies (CISTI), pages 1-4.

Focardi, R., Palmarini, F., Squarcina, M., Steel, G., and Tempesta, M. (2018). Mind your keys? a security evaluation of java keystores. In NDSS.

Gomes, B. D. T. P., Muniz, L. C. M., Da Silva e Silva, F. J., Dos Santos, D. V., Lopes, R. F., Coutinho, L. R., Carvalho, F. O., and Endler, M. (2017). A middleware with comprehensive quality of context support for the internet of things applications. Sensors, 17(12).

Khan, M. A. and Salah, K. (2018). Iot security: Review, blockchain solutions, and open challenges. Future Generation Computer Systems, 82:395-411.

Kortuem, G., Kawsar, F., Sundramoorthy, V., and Fitton, D. (2009). Smart objects as building blocks for the internet of things. IEEE Internet Computing, 14(1):44-51.

Krawczyk, H., Paterson, K. G., and Wee, H. (2013). On the security of the tls protocol: A systematic analysis. In Annual Cryptology Conference, pages 429-448. Springer.

Manzoor, A., Truong, H.-L., and Dustdar, S. (2014). Quality of context: models and applications for context-aware systems in pervasive environments. The Knowledge Engineering Review, 29(2):154-170.

Ngu, A. H., Gutierrez, M., Metsis, V., Nepal, S., and Sheng, Q. Z. (2016). Iot middleware: A survey on issues and enabling technologies. IEEE Internet of Things Journal, 4(1).

Talavera, L. E., Endler, M., Vasconcelos, I., Vasconcelos, R., Cunha, M., and e Silva, F. J. d. S. (2015). The mobile hub concept: Enabling applications for the internet of mobile things. In 2015 IEEE International Conference on Pervasive Computing and Communication Workshops (PerCom Workshops), pages 123-128. IEEE. 